\title{
Predator-prey pattern formation driven by population diffusion based on Moore neighborhood structure
}

\author{
Tousheng Huang ${ }^{1}$, Huayong Zhang ${ }^{1 *}$, Zhengran $\mathrm{Hu}^{1}$, Ge Pan' ${ }^{1}$, Shengnan Ma' ${ }^{1}$ Xiumin Zhang ${ }^{1}$ and \\ Zichun Gao'
}

*Correspondence:

rceens@ncepu.edu.cn

${ }^{1}$ Research Center for Engineering

Ecology and Nonlinear Science,

North China Electric Power

University, Beijing, P.R. China

\section{第 Springer}

\begin{abstract}
Diffusion-driven instability is a basic nonlinear mechanism for pattern formation. Therefore, the way of population diffusion may play a determinative role in the spatiotemporal dynamics of biological systems. In this research, we launch an investigation on the pattern formation of a discrete predator-prey system where the population diffusion is based on the Moore neighborhood structure instead of the von Neumann neighborhood structure widely applied previously. Under pattern formation conditions which are determined by Turing instability analysis, numerical simulations are performed to reveal the spatiotemporal complexity of the system. A pure Turing instability can induce the self-organization of many basic types of patterns as described in the literature, as well as new spiral-spot and labyrinth patterns which show the temporally oscillating and chaotic property. Neimark-Sacker-Turing and flip-Turing instability can lead to the formation of circle, spiral and much more complex patterns, which are self-organized via spatial symmetry breaking on the states that are homogeneous in space and non-periodic in time. Especially, the emergence of spiral pattern suggests that spatial order can generate from temporal disorder, implying that even when the predator-prey dynamics in one site is chaotic, the spatially global dynamics may still be predictable. The results obtained in this research suggest that when the way of population diffusion changes, the pattern formation in the predator-prey systems demonstrates great differences. This may provide realistic significance to explain more general predator-prey coexistence.
\end{abstract}

Keywords: Discrete predator-prey system; Moore neighborhood structure; Pattern formation; Spatiotemporal dynamics; Coupled map lattice

\section{Introduction}

Pattern refers to a heterogeneous macrostructure with certain regularity in space or time, which widely exists in nature, for example, the fish-scale clouds in the sky, the waves on the surface of water, the stripes and spots on the animal's skin, and the regular distribution of populations. The research on nonlinear mechanisms of pattern formation is of great significance for revealing the fundamental laws of natural phenomena. Recently, many researchers have made a great deal of efforts in understanding the pattern self-organization in predator-prey systems [1-7]. Since the predator-prey systems exist in multi-level and

(c) The Author(s) 2019. This article is distributed under the terms of the Creative Commons Attribution 4.0 International License (http://creativecommons.org/licenses/by/4.0/), which permits unrestricted use, distribution, and reproduction in any medium, provided you give appropriate credit to the original author(s) and the source, provide a link to the Creative Commons license, and indicate if changes were made. 
multi-scale in ecosystems, the predator-prey pattern formation reflects the spatiotemporal complexity of ecosystems [8-10] and deserves investigation.

The pattern formation always takes place when a dynamical system is far from a thermodynamic equilibrium state at which the intensive properties of the system are independent of time and no current of matter or energy exists in the interior of the system or at the boundaries with the surroundings [11]. In such a case, the system dynamics often becomes very complex and tends to be heterogeneous. In 1952, Turing [12] established a reaction-diffusion model to explain the patterns on the surface skin of animals and revealed the regularity of spatial pattern formation from mathematics, i.e. the instability of spatial homogeneous stationary state brings spatial symmetry breaking, which results to the self-organization of patterns. The Turing instability theory has been applied in various fields to explain the pattern self-organization phenomena and verified by experiments. In 1991, Ouyang found two-dimensional Turing patterns in experiments [13, 14], which provided evidence for theoretical research results of pattern formation analysis.

In studying the pattern formation of the predator-prey systems, the reaction-diffusion model and Turing instability theory have been widely employed and lots of interesting results have been obtained. For example, Xu et al. investigated the spatiotemporal dynamics of a reaction-diffusion predator-prey system with time delay, determining the effects of spatiotemporal delay and predator rate on the spatiotemporal distributions of the species [2]. Brigatti et al. explored the classical Lotka-Volterra model, which can control the spatial scale of predator-prey interaction range and found the pattern formation of ecosystems via determining the instability which is driven by the range of interaction [3]. Wang et al. studied the spatiotemporal dynamics of a homogeneous diffusive predator-prey system, and proved the existence of non-constant positive steady state solutions [15]. Li found sufficient conditions for the existence of non-constant positive solutions by studying local and global stability of an invasion-diffusion predator-prey system, illustrating the existence possibility of the spatiotemporal pattern [4]. These research works prove the efficiency and effectiveness of the reaction-diffusion model and Turing instability theory.

Until now, the vast majority of research works on predator-prey systems employs continuous predator-prey models. However, over the past decades, more and more researchers realize the discrete properties of the predator-prey systems, such as fragmented habitat, alternation of generations, and so on, and their influence on the system dynamics $[5-7,16]$. Based on the discrete properties, various discrete models have been developed. Generally, the discrete models show power in capturing dynamical characteristics as well as spatiotemporal complexity of the predator-prey systems $[5,17,18]$. In literature, one of the most successful discrete models often applied for studying the spatiotemporal predator-prey dynamics is the coupled map lattice (CML) $[5,17,19-22]$.

The CML describes "a dynamical system with discrete time ('map'), discrete space ('lattice'), and a continuous state" [23]. As described by Kaneko [23], "it usually consists of dynamical elements on a lattice interacting ('coupled') among suitably chosen sets of other elements". This type of model, based on its advantages, is often applied to investigate the pattern formation and spatiotemporal chaos of the predator-prey systems. Via discretizing the reaction-diffusion model, the CML can be developed [16, 17, 22]. And the researchers found that such CMLs are practical in describing nonlinear characteristics and spatiotemporal complexity of predator-prey systems $[16,17]$. Moreover, many new attractive results can be produced with the application of CMLs. Rodrigues et al. [21] revealed 
a rich variety of pattern formation scenarios in a space- and time-discrete predator-prey system with a strong Allee effect and found spatiotemporal multistability under the effects of different initial conditions. Huang et al. researched the discrete predator-prey systems based on CML and revealed complex dynamic transition from non-chaotic state to chaotic state [22]. Also, they studied the spatiotemporal pattern formation of different discrete predator-prey systems, and found the CML can demonstrate new dynamical complexity, including spatiotemporal chaos $[16,17]$.

With its particular nonlinear mechanisms, the CML can further demonstrate and profoundly reveal the spatiotemporal complexity of predator-prey systems. However, the current research works with the application of CML as well as reaction-diffusion model give little attention to the pattern formation under the effects of different ways of population diffusion. In the reaction-diffusion model, the Laplacian operator is mostly often used to represent the population diffusion. Correspondingly in the CML, the discrete form of Laplacian operator is applied, i.e.,

$$
\nabla_{\mathrm{d}}^{2} \phi_{(x, y, t)}=\phi_{(x+1, y, t)}+\phi_{(x-1, y, t)}+\phi_{(x, y+1, t)}+\phi_{(x, y-1, t)}-4 \phi_{(x, y, t)},
$$

in which $\phi$ represents the state variable which is dependent on the space and time variables $x, y, t$. As recorded in the literature, the neighborhood structure used in the discrete Laplacian operator is the so-called von Neumann neighborhood structure. In this research, we turn to study the pattern formation under another type of neighborhood structure, the Moore neighborhood, which is scarcely investigated in the spatiotemporal predator-prey models, including both continuous reaction-diffusion models and CML models. The discrete Laplacian operator based on Moore neighborhood structure can be expressed as

$$
\begin{aligned}
\nabla_{\mathrm{d}}^{2} \phi_{(x, y, t)}= & \phi_{(x+1, y, t)}+\phi_{(x-1, y, t)}+\phi_{(x, y+1, t)}+\phi_{(x, y-1, t)}+\phi_{(x+1, y-1, t)}+\phi_{(x+1, y+1, t)} \\
& +\phi_{(x-1, y-1, t)}+\phi_{(x-1, y+1, t)}-8 \phi_{(x, y, t)} .
\end{aligned}
$$

The feasibility of applying Moore neighborhood structure for population diffusion can be described in the following aspects. First, the original continuous Laplacian operator, if considered separately in two-dimensional space, describes the diffusion around located site in the form of waving circles. Intuitively, such encircling type of diffusion is better exhibited by the Moore neighborhood than the von Neumann neighborhood, which is a numerical approximation of the continuous Laplacian operator. Second, the population diffusion with Moore neighborhood structure shows higher spatial extension than that described by von Neumann neighborhood structure. Since the Moore neighborhood structure allows the predator and prey spreading to any site around, the population diffusion is isotropic, i.e., the spatial dispersal of the predator and the prey populations can extend to all sites around by arbitrary ways. Therefore, the Laplacian operator with Moore neighborhood structure actually describes a case of population diffusion which is not restrained by external factors, such as topography, landscape, and so on. Third, dependent on the distribution and connection of discrete habitat patches in natural space, different neighborhood structures for population diffusion should be applied. The Moore neighborhood structure suggests that the discrete habitat patches compose of a complex network, where each patch is connected to eight adjacent patches. Based on the complex network, the predator-prey system exhibits population diffusion among these "adjacent" patches. 
Consequently, the Moore neighborhood structure holds significance from the perspectives of ecology and complex network.

In this research, we concern the pattern formation of a modified Leslie-Gower predator-prey system under the influence of population diffusion in expressing as (2). The Leslie-Gower predator-prey system is one of the important systems in population dynamics. The Leslie-Gower functional response was initially built and studied by Leslie and Gower, who assumed that interacting species grow according to the logistic law and that the environmental carrying capacity for the predator is not a constant but proportional to the population size of the prey [24]. However, due to the rarity of the prey, the predator can switch over to other food, but its growth is still limited by the fact that its favorite prey is not available in abundance [24-26]. Based on such case, a modified Leslie-Gower functional response was further developed and extensively studied by many researchers [27-30]. With the CML and Moore neighborhood structure considered in this research, new spatiotemporal complexity will be revealed for such a predator-prey system.

The organization of this research work is arranged as follows. Section 2 will establish a discrete predator-prey model which is given by a CML where Moore neighborhood structure is applied for population diffusion. Section 3 will perform Turing instability analysis, and determine the pattern formation conditions of the predator-prey system. In Sect. 4, spatial patterns induced by Turing instability will be numerically simulated and displayed to show the spatiotemporal complexity of the modified Leslie-Gower predator-prey system. And finally, Sect. 5 will provide the discussion and conclusions.

\section{Model development}

The CML can be developed from the discretization of the continuous dynamical model. Therefore, the model development in this research starts from a continuous spatiotemporal predator-prey model which is governed by partial differential equations. Here, we consider a modified Leslie-Gower predator-prey system with Holling-type II functional response [31]. The corresponding equations for the predator-prey model are described as [31]

$$
\begin{aligned}
& \frac{d U}{d T}=\left(a_{1}-b_{1} U-\frac{c_{1} V}{U+k_{1}}\right) U+D_{1}\left(\frac{\partial^{2} U}{\partial X^{2}}+\frac{\partial^{2} U}{\partial Y^{2}}\right), \\
& \frac{d V}{d T}=\left(a_{2}-\frac{c_{2} V}{U+k_{2}}\right) V+D_{2}\left(\frac{\partial^{2} V}{\partial X^{2}}+\frac{\partial^{2} V}{\partial Y^{2}}\right),
\end{aligned}
$$

where $U$ and $V$ are the densities of prey and predator, respectively; $T$ stands for time and $(X, Y)$ represents the spatial position of the species when they move in a two-dimensional space; $a_{1}$ and $a_{2}$ describe the growth rate of the prey and the predator; $b_{1}$ measures the strength of competition among individuals of the prey; $c_{1}$ is the maximum value of the per capita reduction of the prey due to the predator; $k_{1}$ measures the extent to which environment provides protection to the prey; $c_{2}$ and $k_{2}$ have similar meaning to $c_{1}$ and $k_{1}$ relatively to the predator; $D_{1}$ and $D_{2}$ are the diffusion coefficients of prey and predator, respectively.

The spatiotemporal predator-prey model with modified Leslie-Gower and Hollingtype II functional responses is well recognized in the literature and has been studied [24- 
26, 32]. With the following scaling transformations:

$$
\begin{array}{llrl}
t=a_{1} T, & u=\frac{b_{1}}{a_{1}} H, & v=\frac{c_{2} b_{1}}{a_{1} a_{2}} P, & x=\sqrt{\frac{a_{1}}{D_{1}}} X,
\end{array}
$$

Eqs. (3a)-(3b) can be changed into the following simplified form:

$$
\begin{aligned}
& \frac{d u}{d t}=u(1-u)-\frac{a u v}{u+e_{1}}+\nabla^{2} u, \\
& \frac{d v}{d t}=b v\left(1-\frac{v}{u+e_{2}}\right)+\delta \nabla^{2} u .
\end{aligned}
$$

Via discretizing Eqs. (5a)-(5b), the new CML model is developed. First we consider a two-dimensional rectangular domain including $n \times n$ grid cells, with time interval and space interval given as $\tau$ and $h$. Each grid cell represents a spatial site where the predator and prey dwell. For simplification, we still use $x, y, t$ to represent the discrete space and time variables. Then we define two state varaibles, $u_{(x, y, t)}$ and $v_{(x, y, t)}(x, y \in\{1,2,3, \ldots, n\}$ and $\left.t \in Z^{+}\right)$, representing the prey density and the predator density in the $(x, y)$ site at the time $t$. According to the previous literature [16, 17, 22], in developing the CML, the ecological processes of the predator-prey system are often segregated into two distinctly different stages at each discrete step from $t$ to $t+1$, namely dispersal stage and reaction stage. The dispersal stage includes the spatial diffusion of the prey and the predator in space, whereas the reaction stage includes the growth and death of the prey and the predator, and the predation between the two populations. Through discretizing the spatial terms of Eqs. (5a)-(5b), the dispersal stage can be expressed as follows:

$$
\begin{aligned}
& u_{(x, y, t)}^{\prime}=u_{(x, y, t)}+\frac{\tau}{h^{2}} \nabla_{\mathrm{d}}^{2} u_{(x, y, t)}, \\
& v_{(x, y, t)}^{\prime}=v_{(x, y, t)}+\frac{\tau}{h^{2}} \delta \nabla_{\mathrm{d}}^{2} v_{(x, y, t)},
\end{aligned}
$$

where $\nabla_{\mathrm{d}}^{2}$ denotes the discrete Laplacian operator. Here, we utilize the Moore neighborhood structure. Correspondingly, the expressions of $\nabla_{d}^{2} u_{(x, y, t)}$ and $\nabla_{d}^{2} v_{(x, y, t)}$ are given by Eq. (2).

Through discretizing the non-spatial terms of Eqs. (5a)-(5b), the reaction stage of the CML can be obtained:

$$
\begin{aligned}
& u_{(x, y, t+\tau)}=f_{1}\left(u_{(x, y, t)}^{\prime}, v_{(x, y, t)}^{\prime}\right), \\
& v_{(x, y, t+\tau)}=g_{1}\left(u_{(x, y, t)}^{\prime}, v_{(x, y, t)}^{\prime}\right),
\end{aligned}
$$

where $f_{1}$ and $g_{1}$ are functions determined by the local interaction of the predator-prey system. The two functions are expressed by [31, 33, 34]

$$
\begin{aligned}
& f_{1}(u, v)=u+\tau\left(u(1-u)-\frac{a v u}{u+e_{1}}\right) \\
& g_{1}(u, v)=v+\tau b v\left(1-\frac{v}{u+e_{2}}\right)
\end{aligned}
$$


Generally in the literature, the dispersal stage is considered to occur before the reaction stage in each iteration. Combining the above equations, the expression of the CML is obtained:

$$
\begin{aligned}
& u_{(x, y, t+\tau)}=f_{1}\left(u_{(x, y, t)}+\frac{\tau}{h^{2}} \nabla_{\mathrm{d}}^{2} u_{(x, y, t)}, v_{(x, y, t)}+\frac{\tau}{h^{2}} \delta \nabla_{\mathrm{d}}^{2} v_{(x, y, t)}\right), \\
& v_{(x, y, t+\tau)}=g_{1}\left(u_{(x, y, t)}+\frac{\tau}{h^{2}} \nabla_{\mathrm{d}}^{2} u_{(x, y, t)}, v_{(x, y, t)}+\frac{\tau}{h^{2}} \delta \nabla_{\mathrm{d}}^{2} v_{(x, y, t)}\right),
\end{aligned}
$$

which describes a spatiotemporally discrete predator-prey system. The initial condition and boundary condition for the CML are given as follows:

$$
\begin{array}{ll}
u_{(x, y, 0)}=u_{0}(1+0.1(\varsigma-0.5)), \quad v_{(x, y, 0)}=v_{0}(1+0.1(\varsigma-0.5)), \\
u_{(0, y, t)}=u_{(n, y, t)}, & u_{(1, y, t)}=u_{(n+1, y, t)}, \\
u_{(x, 0, t)}=u_{(x, n, t)}, & u_{(x, 1, t)}=u_{(x, n+1, t)}, \\
v_{(0, y, t)}=v_{(n, y, t)}, & v_{(1, y, t)}=v_{(n+1, y, t)}, \\
v_{(x, 0, t)}=v_{(x, n, t)}, & v_{(x, 1, t)}=v_{(x, n+1, t)},
\end{array}
$$

where $u_{0}$ and $v_{0}$ are fixed values determined by the stable fixed point and $\varsigma$ follows standard uniform distribution. From the ecological point of view, all the parameters involved in the CML model are positive and the values of the state variables are nonnegative.

\section{Stability analysis}

\subsection{Local stability analysis}

First the spatially homogeneous stationary states of the discrete predator-prey system need to be obtained. According to the spatial homogeneity of the discrete system, we have

$$
\nabla_{\mathrm{d}}^{2} u_{(x, y, t)}=0, \quad \nabla_{\mathrm{d}}^{2} v_{(x, y, t)}=0 .
$$

Substituting Eq. (11) into the expression of the CML, the dynamics of the system turns to be

$$
\begin{aligned}
& u_{(x, y, t+\tau)}=u_{(x, y, t)}+\tau u_{(x, y, t)}\left(1-u_{(x, y, t)}-\frac{a v_{(x, y, t)}}{u_{(x, y, t)}+e_{1}}\right), \\
& v_{(x, y, t+\tau)}=v_{(x, y, t)}+b \tau v_{(x, y, t)}\left(1-\frac{v_{(x, y, t)}}{u_{(x, y, t)}+e_{2}}\right) .
\end{aligned}
$$

Let $u_{(x, y, t+\tau)}=u_{(x, y, t)}$, and $v_{(x, y, t+\tau)}=v_{(x, y, t)}$ (notice that these two equations establish for any $x$ and $y$ ), and solving Eqs. (12a)-(12b), then the fixed points of the system can be obtained [22]:

$$
\left(u_{1}, v_{1}\right):(0,0), \quad\left(u_{2}, v_{2}\right):\left(1, e_{2}\right), \quad\left(u_{3}, v_{3}\right):(1,0), \quad\left(u_{4}, v_{4}\right):\left(u^{*}, v^{*}\right)
$$

where

$$
\begin{aligned}
& u^{*}=\frac{1}{2}\left(1-a-e_{1}+\sqrt{\left(a+e_{1}-1\right)^{2}-4\left(a e_{2}-e_{1}\right)}\right), \\
& v^{*}=u^{*}+e_{2} .
\end{aligned}
$$


The sufficient and necessary conditions for that $\left(u^{*}, v^{*}\right)$ is positive are

$$
\begin{aligned}
& \left(a+e_{1}-1\right)^{2}-4\left(a e_{2}-e_{1}\right)>0, \\
& e_{1}>\max \left(1-a, a e_{2}\right) \quad \text { or } \quad e_{1}<1-a .
\end{aligned}
$$

The local stability of the fixed point is determined with application of the method of Jacobian matrix. Since the pattern formation needs the nontrivial homogeneous stationary state, which is corresponding to the fixed point $\left(u^{*}, v^{*}\right)$, we just concern the stability of $\left(u^{*}, v^{*}\right)$. The Jacobian matrix associated to Eqs. $(12 \mathrm{a})-(12 \mathrm{~b})$ at any point is described as

$$
J(u, v)=\left(\begin{array}{cc}
1+\tau\left(1-2 u-\frac{a v e_{1}}{\left(u+e_{1}\right)^{2}}\right) & -\frac{\tau a u}{u+e_{1}} \\
\frac{\tau b v^{2}}{\left(u+e_{2}\right)^{2}} & 1+\tau\left(b-\frac{2 b v}{u+e_{2}}\right)
\end{array}\right) .
$$

Substituting the value of $\left(u^{*}, v^{*}\right)$ into (16), we get

$$
A=J\left(u^{*}, v^{*}\right)=\left(\begin{array}{ll}
a_{11} & a_{12} \\
a_{21} & a_{22}
\end{array}\right)
$$

where

$$
\begin{aligned}
& a_{11}=1+\tau\left(1-2 u^{*}-\frac{a\left(u^{*}+e_{2}\right) e_{1}}{\left(u^{*}+e_{1}\right)^{2}}\right), \quad a_{12}=-\frac{\tau a u^{*}}{u^{*}+e_{1}}, \\
& a_{21}=\tau b, \quad a_{22}=1-\tau b .
\end{aligned}
$$

The two eigenvalues of matrix (17) are

$$
\lambda_{1,2}=\frac{1}{2}\left(-p \pm \sqrt{p^{2}-4 q}\right)
$$

where

$$
p=a_{11}+a_{22}, \quad q=a_{11} a_{22}-a_{12} a_{21} .
$$

According to the criterion of stability of the fixed point, if $\left|\lambda_{1}\right|<1$ and $\left|\lambda_{2}\right|<1$, the corresponding fixed point is considered to be stable. As shown in previous research [16], this criterion of stability is equivalent to $q<1,-(1+q)<p<1+q$. Through calculations, the conditions for $\left(u^{*}, v^{*}\right)$ to be stable are determined as

$$
\begin{aligned}
& (1-\tau b) B+(\tau a-1) b A^{2}-\tau a b e_{1} A<0, \\
& B-a A^{2}+a e_{1} A<0, \\
& \tau(\tau b-2) B-\left(\tau^{2} a b+2 \tau b+4\right) A^{2}+\tau^{2} a b e_{1} A<0,
\end{aligned}
$$

where $A=\frac{1}{2}\left(1-a+e_{1}+\sqrt{\left(a+e_{1}-1\right)^{2}-4\left(a e_{2}-e_{1}\right)}\right)$ and $B=a e_{1}\left(e_{1}-e_{2}\right)-a e_{1} A+(1+$ $\left.2 e_{1}\right) A^{2}-2 A^{3}$. 


\subsection{Turing instability analysis}

Turing instability, also known as diffusion-driven instability, occurs when a spatially homogeneous stationary state that would be stable in the absence of diffusion is destabilized by diffusion $[35,36]$. The Turing instability always takes place along with the spatial symmetry breaking which shows the spatiotemporal dynamical system turning from a state with spatial invariance to another state that is spatially variant under the broken symmetry, suggesting the change from spatially homogeneous states to Turing patterns. Generally, the occurrence of a Turing instability needs the satisfaction of two conditions. First, the system has a nontrivial stable spatially homogeneous stationary state. Second, this state is unstable to spatially heterogeneous perturbations. Under the influence of Turing instability, local spatially heterogeneous perturbations on the stable homogeneous state can gradually expand to the global spatial domain.

On the basis of the stable fixed point $\left(u^{*}, v^{*}\right)$, the Turing instability is then analyzed. Considering a small spatially heterogeneous perturbation $\varepsilon_{(x, y, t)}$ and $\sigma_{(x, y, t)}$ on $\left(u^{*}, v^{*}\right)$, we have

$$
u_{(x, y, t)}=u^{*}+\varepsilon_{(x, y, t)}, \quad v_{(x, y, t)}=v^{*}+\sigma_{(x, y, t)} .
$$

Substituting (22) into Eqs. (9a)-(9a) and keeping the linear-order terms yield difference equations for $\varepsilon_{(x, y, t)}$ and $\sigma_{(x, y, t)}$,

$$
\begin{aligned}
& \varepsilon_{(x, y, t+\tau)}=a_{11}\left(\varepsilon_{(x, y, t)}+\frac{\tau}{h^{2}} \nabla_{\mathrm{d}}^{2} \varepsilon_{(x, y, t)}\right)+a_{12}\left(\sigma_{(x, y, t)}+\frac{\tau}{h^{2}} \delta \nabla_{\mathrm{d}}^{2} \sigma_{(x, y, t)}\right), \\
& \sigma_{(x, y, t+\tau)}=a_{21}\left(\varepsilon_{(x, y, t)}+\frac{\tau}{h^{2}} \nabla_{\mathrm{d}}^{2} \varepsilon_{(x, y, t)}\right)+a_{22}\left(\sigma_{(x, y, t)}+\frac{\tau}{h^{2}} \delta \nabla_{\mathrm{d}}^{2} \sigma_{(x, y, t)}\right) .
\end{aligned}
$$

The Fourier-type generic solution of the system of difference equations (23a)-(23b) can be given as

$$
\left(\begin{array}{c}
\varepsilon_{(x, y, t)} \\
\sigma_{(x, y, t)}
\end{array}\right)=\left(\begin{array}{c}
\bar{\varepsilon}_{t} \\
\bar{\sigma}_{t}
\end{array}\right) \cos \left(q_{1} x\right) \cos \left(q_{2} y\right),
$$

where $\bar{\varepsilon}_{t}$ and $\bar{\sigma}_{t}$ are new variables independent of space, $q_{1}$ and $q_{2}$ are wavenumbers and unknown. Substituting the Fourier-type generic solution (24) into Eqs. (23a)-(23b) leads to the following results:

$$
\begin{aligned}
\bar{\varepsilon}_{t+\tau}= & a_{11}\left(\bar{\varepsilon}_{t}+\frac{2 \tau}{h^{2}}\left(\cos q_{1}+\cos q_{2}+2 \cos q_{1} \cos q_{2}-4\right) \bar{\varepsilon}_{t}\right) \\
& +a_{12}\left(\bar{\sigma}_{t}+\frac{2 \tau}{h^{2}} \delta\left(\cos q_{1}+\cos q_{2}+2 \cos q_{1} \cos q_{2}-4\right) \bar{\sigma}_{t}\right), \\
\bar{\sigma}_{t+\tau}= & a_{21}\left(\bar{\varepsilon}_{t}+\frac{2 \tau}{h^{2}}\left(\cos q_{1}+\cos q_{2}+2 \cos q_{1} \cos q_{2}-4\right) \bar{\varepsilon}_{t}\right) \\
& +a_{22}\left(\bar{\sigma}_{t}+\frac{2 \tau}{h^{2}} \delta\left(\cos q_{1}+\cos q_{2}+2 \cos q_{1} \cos q_{2}-4\right) \bar{\sigma}_{t}\right) .
\end{aligned}
$$


Let

$$
\begin{aligned}
& k_{1}=1+\frac{2 \tau}{h^{2}}\left(\cos q_{1}+\cos q_{2}+2 \cos q_{1} \cos q_{2}-4\right), \\
& k_{2}=1+\frac{2 \tau}{h^{2}} \delta\left(\cos q_{1}+\cos q_{2}+2 \cos q_{1} \cos q_{2}-4\right) .
\end{aligned}
$$

It should be noticed that $k_{1}$ and $k_{2}$ are particularly corresponding to the Moore neighborhood structure. For other neighborhood structures, these expressions are different since each grid cell included in the neighborhood has special characteristics under the Fourier expansion. For the von Neumann neighborhood structure, $k_{1}$ and $k_{2}$ change to be $k_{1}=1+\frac{2 \tau}{h^{2}}\left(\cos q_{1}+\cos q_{2}-2\right), k_{2}=1+\frac{2 \tau}{h^{2}} \delta\left(\cos q_{1}+\cos q_{2}-2\right)$.

The Jacobian matrix of difference equations (25a)-(25b) can be written as

$$
J^{*}=\left(\begin{array}{ll}
a_{11} k_{1} & a_{12} k_{2} \\
a_{21} k_{1} & a_{22} k_{2}
\end{array}\right) .
$$

According to the Turing instability conditions as recorded in the literature, when the solutions of the difference equations (25a)-(25b) diverge, Turing instability takes place in the discrete predator-prey system. It means the occurrence of a Turing instability needs that the modulus of one of the eigenvalues of matrix (27) is larger than one. The two eigenvalues of (27) are calculated as

$$
\bar{\lambda}_{ \pm}=\frac{1}{2}\left(k_{1} a_{11}+k_{2} a_{22} \pm \sqrt{\left(k_{1} a_{11}+k_{2} a_{22}\right)^{2}-k_{1} k_{2} \operatorname{det} A}\right) .
$$

That $\left|\bar{\lambda}_{+}\right|$or $\left|\bar{\lambda}_{-}\right|$is larger than 1 leads to a necessary condition for Turing instability, which can be given as

$$
\left|k_{1} a_{11}+k_{2} a_{22}\right|>1+k_{1} k_{2} \operatorname{det} A
$$

Under this Turing instability condition (29), the stable state $\left(u^{*}, v^{*}\right)$ is destabilized and the predator-prey system converges to a new state which maintains heterogeneous in space. This means the Turing instability induces pattern formation. From the above calculations, two cases of Turing instability conditions can be obtained. If we have $\max \left(\bar{\lambda}_{+}, \bar{\lambda}_{-}\right)>1$, it suggests the occurrence of diffusion-driven plus-one bifurcation; if we have $\min \left(\bar{\lambda}_{+}, \bar{\lambda}_{-}\right)<-1$, it implies the emergence of diffusion-driven minus-one bifurcation. The conditions for these cases of Turing instability are described as follows:

(1) A diffusion-driven plus-one bifurcation occurs when

$$
\begin{aligned}
& |\operatorname{Tr} A|<1+\operatorname{det} A, \\
& \operatorname{det} A<1, \\
& 1-\left(k_{1} a_{11}+k_{2} a_{22}\right)+k_{1} k_{2} \operatorname{det} A<0 ;
\end{aligned}
$$

(2) a diffusion-driven minus-one bifurcation occurs when inequalities (30a) and (30b) hold, but (30c) turns into

$$
1+\left(k_{1} a_{11}+k_{2} a_{22}\right)+k_{1} k_{2} \operatorname{det} A<0 .
$$




\section{Pattern formation}

The Turing instability analysis gives the conditions for pattern formation of the discrete predator-prey system. Based on the pattern formation conditions, numerical simulations are performed to demonstrate the predator-prey patterns. For the simulations, the parameter values are given based on former research work [30]. In this research, we provide a group of feasible parameter ranges as: $a=0.89 \sim 1.35, b=0.1781 \sim 1.21, e_{1}=0.3 \sim 0.31$, $e_{2}=0.2 \sim 0.21, \delta=1 \sim 30, h=6 \sim 15, \tau=0.2 \sim 3.64$. Within these parameter ranges, the parameter values should satisfy two aspects of conditions. First, to ensure non-negativity of $u$ and $v$ in the numerical simulations, the value of $\delta \tau / h^{2}$ must be less than $0.5[16,37]$. Second, the values of parameters $a, b, e_{1}, e_{2}, \delta, h$ and $\tau$ should satisfy conditions (30a)(30c) and (31), implying Turing instability. Additionally, the value of parameter $n$ which reflects the domain size of the space would be better ranging in $50 \sim 400[5-7,16,17$, $21,22,31,36]$. A too small value of $n$ would lead to simulation results of no significance, whereas a too large value of $n$ brings about an excessively long simulation running time. Notice that the Moore neighborhood of the grid cell at the boundary is adjusted according to periodic boundary condition. In this research, the value of $n$ is given as 50, 100 and 200 . The change of $n$ value does not influence the pattern types in simulations and is merely to demonstrate the patterns in the best window.

The initial condition for pattern simulation is given by applying small random perturbations on the stable homogeneous stationary state as described in Eq. (10a). By changing different parameter values, we get a series of spatial patterns that reflect different dynamic behaviors of the predator and the prey. Since the spatial patterns of predator and prey always display similar or complementary configurations, we can choose one of them (in this research the prey pattern is chosen) to make exhibition, description and analysis. Notice that the red color represents a high prey density, whereas the blue color shows a low prey density.

Figure 1 displays the classical patterns at various values of parameter $a$ under the pure Turing instability mechanism, with the other parameters fixed as $e_{1}=0.3, e_{2}=0.2, b=$ $0.1781, \delta=30, h=6, \tau=0.2$. It can be found that the change of the maximum consumption rate of the prey consumed by the predator can induce the transition of patterns in the discrete system. With the increase of the $a$ value from 0.89 to 1.35 , the spatial pattern gradually transforms to a cold spot shape from spatially homogeneous state (Fig. 1a and $1 b)$, then the cold spot-stripe pattern, stripe pattern and hot spot-stripe pattern appear one after another (Fig. 1c, 1d and 1e), and finally the system stays at the hot spot pattern (Fig. 1f). Such pattern transition process may result from that the consumption ability of predator to prey enlarges the predator population and consequently the range of activities of the prey individuals is reduced. The continuous shrinking of the range of prey activities leads to the change of pattern shapes.

More complex pattern transition can be induced by the variation of two or more parameters. As shown in Fig. 2, a distribution of spatial patterns in the $a-b$ parameter space is demonstrated. It is found that each type of spatial patterns, denoted by different marks, occupies a specific area in the region diagram of parameters $a$ and $b$. Figure 2 also reveals that with the change of parameter $a$, the discrete predator-prey system always exhibits a pattern transition process as displayed in Fig. 1 . The change of parameter $b$ mainly influences the borders for each pattern areas, and therefore may generate the pattern transition between the adjacent areas, such as the transitions between cold spot and cold spot-stripe 


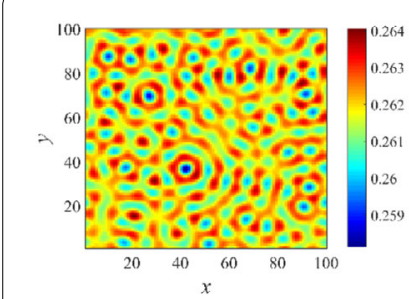

(a) $a=0.898$

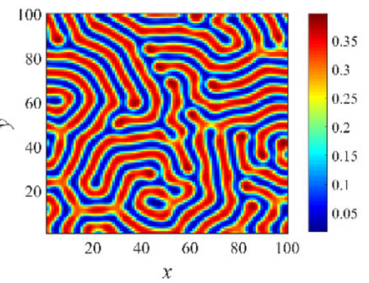

(d) $a=1.05$

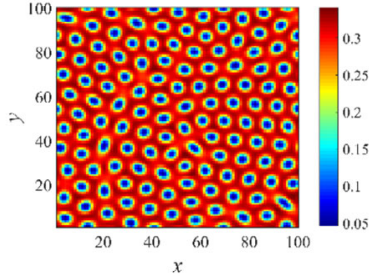

(b) $a=0.93$

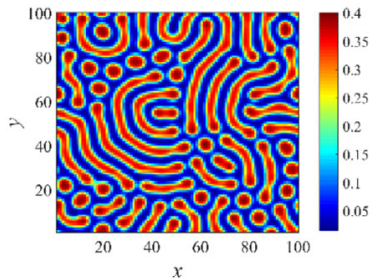

(e) $a=1.13$

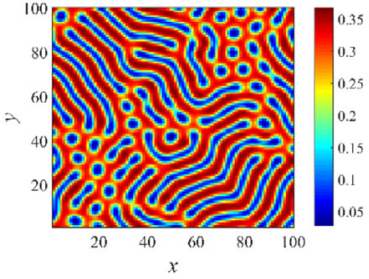

(c) $a=0.98$

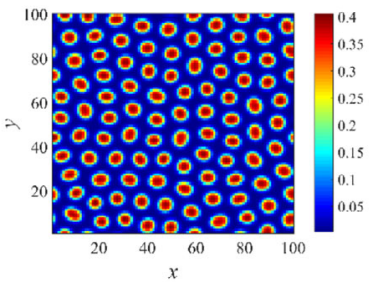

(f) $a=1.35$

Figure 1 Various self-organized patterns induced by Turing instability under different $a$ values, (a) approximately homogeneous pattern, (b) cold spot pattern, (c) cold spot-stripe pattern, (d) stripe pattern, (e) hot spot-stripe pattern, (f) hot spot pattern. The other parameter values are given as $e_{1}=0.3, e_{2}=0.2$, $b=0.1781, \delta=30, h=6, \tau=0.2, n=100, t=10,000$

Figure 2 Distribution of different types of patterns in a region diagram of parameters $a$ and $b$. The meaning of marks are described as follows: eight-pointed stars for homogeneous stationary state, five-pointed stars for approximately homogeneous patterns, inverted triangles for cold spot patterns, triangles for cold spot-stripe patterns, diamonds for stripe patterns, dots for hot spot-stripe patterns, and squares for hot spot patterns. $e_{1}=0.3$, $e_{2}=0.2, \delta=30, h=6, \tau=0.2, n=200, t=10,000$

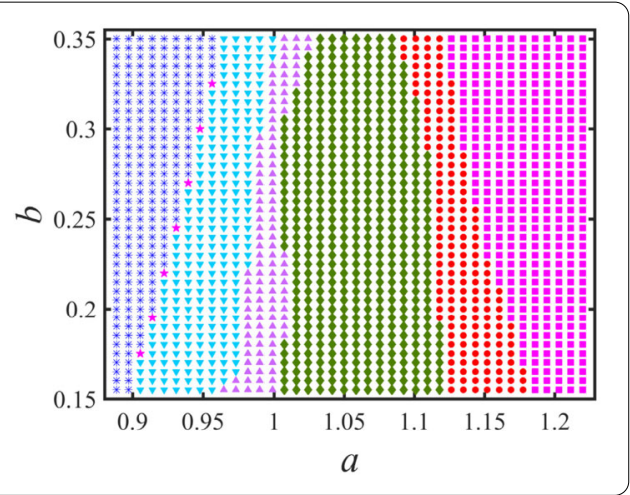

patterns, and between hot spot-stripe and hot spot patterns. Via comparison, we find that the change of parameter $a$ which measures the consumption ability of predator to prey is more sensible than the change of parameter $b$ which measures the growth rate of the predator population to produce the variation of patterns, i.e., to shift the way for coexistence of the predator and the prey.

The pattern types as displayed in Fig. 1 are common in predator-prey systems. However, when the population diffusion is based on the Moore neighborhood structure, new different patterns can be exhibited, as shown in the following figures. Figure 3 displays the evolving process of a spiral-spot pattern. We start from randomly perturbing the homogeneous stationary state $\left(u^{*}, v^{*}\right)$. As the time grows, the predator-prey system experiences disordered transient states (Fig. 3a-b), and then spiral patches emerge in the pattern, alternating with vacant areas of no prey individuals (Fig. 3c-d). Moreover, the prey pattern shows a "second-order" structure: if we consider the spiral patches and vacant areas as background, hot spots of prey patches distribute all around in the pattern. It is also found that the prey pattern in Fig. 3 cannot reach static state but is always fluctuating among 


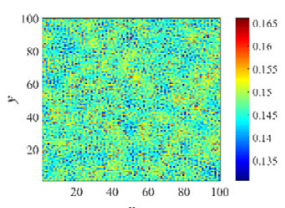

(a)

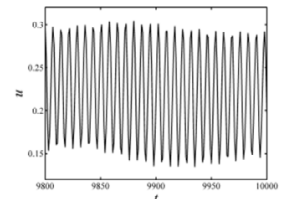

(e)

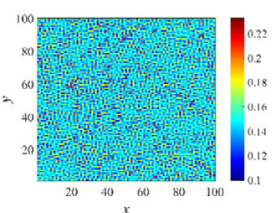

(b)

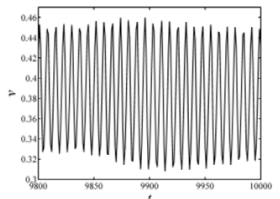

(f)

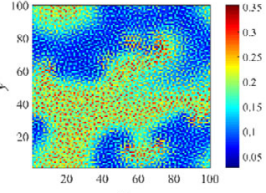

(c)

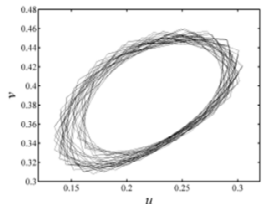

(g)

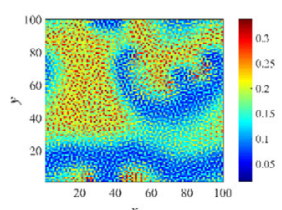

(d)

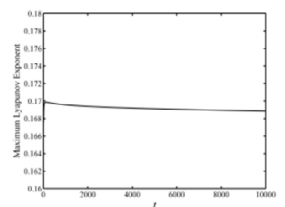

(h)

Figure 3 Self-organziation and nonlinear properties of a spiral-spot pattern. (a)-(d) Evolution of the prey pattern at transient times $t=10, t=100, t=1000$, and $t=10,000$, respectively; $(\mathbf{e})-(\mathbf{g})$ wave diagrams and phase portrait of the prey and the predator in a random site of the predator and prey patterns; (h) maximum Lyapunov exponent diagram corresponding to the prey pattern. The parameter values are given as $a=1.1$, $e_{1}=0.3, e_{2}=0.2, b=0.3739, \tau=2.828, \delta=3.2, h=8, n=100$

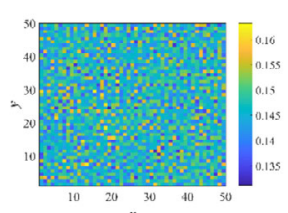

(a)

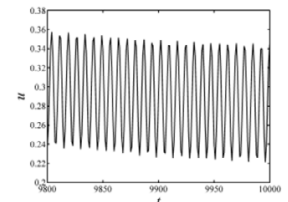

(e)

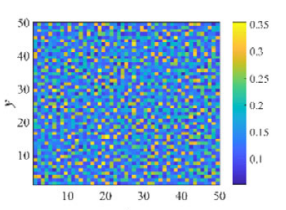

(b)

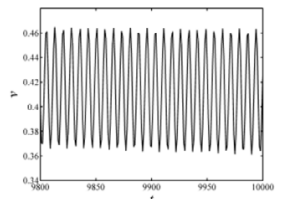

(f)

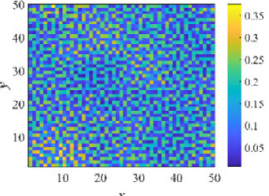

(c)

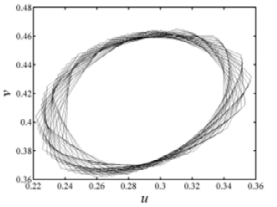

(g)

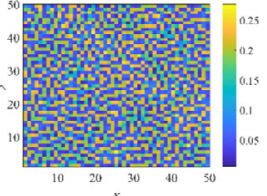

(d)

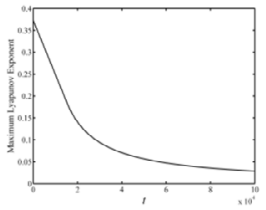

(h)

Figure 4 Self-organziation and nonlinear properties of a labyrinth pattern when the parameter values are given as $a=1.1, e_{1}=0.3, e_{2}=0.2, b=0.3739, \tau=2.36, \delta=4, h=8, n=50$. (a)-(d) Evolution of the prey pattern at transient times $t=10, t=100, t=500$, and $t=1000$, respectively; $(\mathbf{e})-(\mathbf{g})$ wave diagrams, phase portrait and maximum Lyapunov exponent diagram which present same nonlinear properties like in Fig. 3

similar configurations. The fluctuation of prey pattern can be verified by Fig. 3e-g, which demonstrates the oscillation of the predator and prey densities along the time. Figure $3 \mathrm{~h}$ shows a positive maximum Lyapunov exponent, suggesting the self-organized pattern is spatiotemporally chaotic in the oscillation.

Figures 4 and 5 demonstrate the self-organization and the nonlinear properties of two labyrinth patterns. Labyrinth patterns are common in the predator-prey systems and were frequently found in the numerical simulations for predator-prey patterns. However, when compared with former numerical research, the labyrinth patterns here exhibit new configurations, which reflect particular structures for the spatial distribution of prey population under the diffusion based on the Moore neighborhood structure. These patterns may explain the complicated labyrinths for the animals burrowing into the ground after a long evolution under the predator-prey relationships. As suggested by the wave diagrams, phase portraits and positive Lyapunov exponents in Figs. 4 and 5, we find the two 


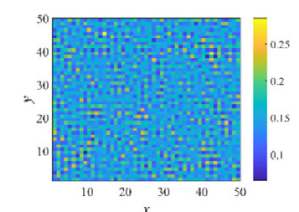

(a)

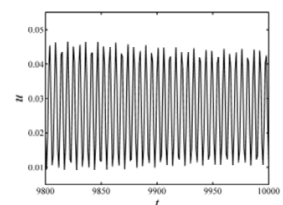

(e)

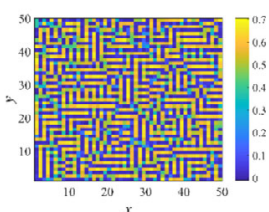

(b)

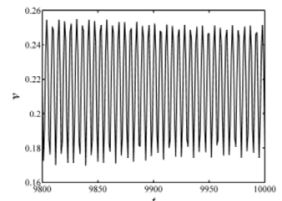

(f)

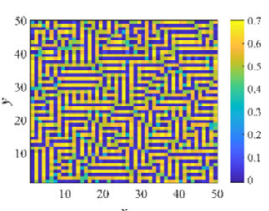

(c)

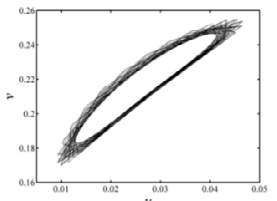

(g)

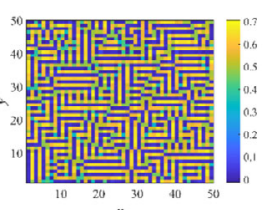

(d)

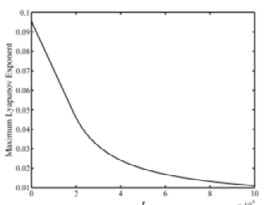

(h)

Figure 5 Self-organziation and nonlinear properties of a labyrinth pattern when the parameter values are given as $a=1.1, e_{1}=0.3, e_{2}=0.2, b=0.38, \tau=2.5, \delta=10, h=15, n=50$. (a)-(d) Evolution of the prey pattern at transient times $t=10, t=30, t=100$, and $t=1000$, respectively; (e)-(g) wave diagrams, phase portrait and maximum Lyapunov exponent diagram which present same nonlinear properties like in Fig. 3

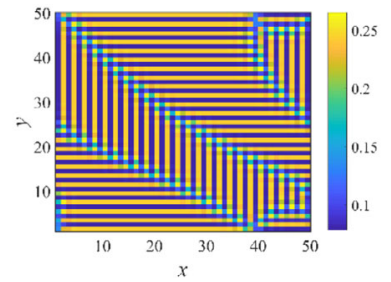

(a) $\tau=1.34$

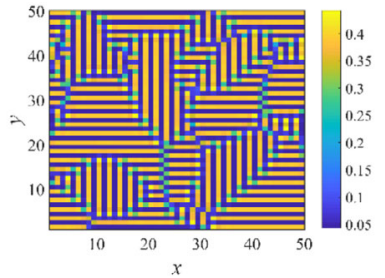

(b) $\tau=1.77$

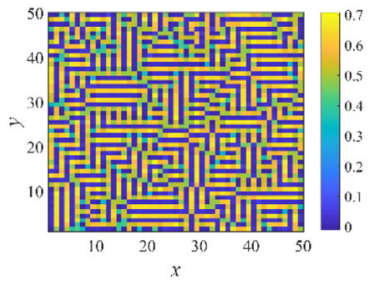

(c) $\tau=2.5$

Figure 6 A transition from stripe pattern to labyrinth pattern with the increase of parameter $\tau$. The other parameter values are given as $a=1.1, e_{1}=0.3, e_{2}=0.2, b=0.38, \delta=10, h=15, n=50$

labyrinth patterns show the properties of temporal oscillation and spatiotemporal chaos. In comparison with the stationary labyrinth patterns produced by the diffusion under von Neumann neighborhood structure, the dynamic properties of oscillating labyrinth patterns imply the predator and prey are quite active in space and time driven by a higher efficiency of diffusion.

Figure 6 shows a transition process of the prey pattern with the change of parameter $\tau$. This transition process suggests the origination of labyrinth pattern from stripe pattern. As demonstrated in Fig. 6, the consequence for the increase of $\tau$ value is the continual fragmentation of the stripes in the pattern. Therefore, as the long stripes are broken into many small short stripes with different directions, a transition from stripe pattern to labyrinth pattern can be observed.

The patterns demonstrated in the above figures are self-organized under the mechanism of pure Turing instability. Furthermore, the predator-prey system may also generate Neimark-Sacker instability, which is induced by Neimark-Sacker bifurcation, leading to the destabilization of the stable state $\left(u^{*}, v^{*}\right)$ and the occurrence of a new state that is homogeneous in space but quasiperiodically oscillating in time. When the discrete predatorprey system undergoes Neimark-Sacker instability and Turing instability simultaneously, spiral or circle patterns may be present, as shown in Figs. 7-8. In order to better display the self-organization of a circle pattern, Fig. 7 is plotted in a three-dimensional image. With 


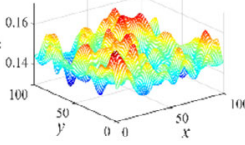

(a)

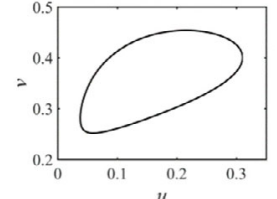

(e)

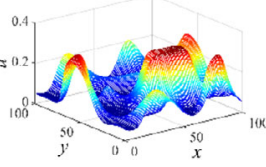

(b)

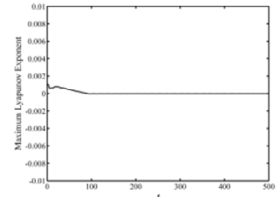

(f)

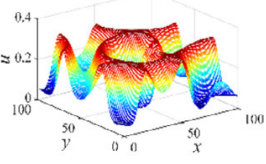

(c)

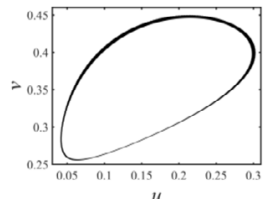

(g)

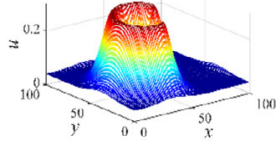

(d)

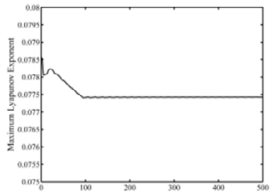

(h)

Figure 7 Self-organziation and nonlinear properties of a circle pattern. (a)-(d) Evolution of the prey pattern at transient times $t=100, t=1000, t=3000$, and $t=5000$, respectively; $(\mathbf{e})-(\mathbf{h})$ phase portraits and maximum Lyapunov exponent diagrams in the cases of uncoupling and coupling the population diffusion. The parameter values are given as $a=1.1, e_{1}=0.3, e_{2}=0.2, b=0.2, \tau=1.89, \delta=2, h=10, n=100$

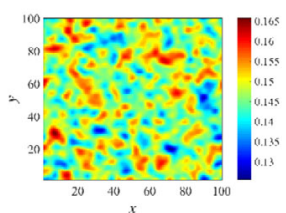

(a)

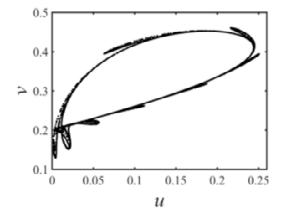

(e)

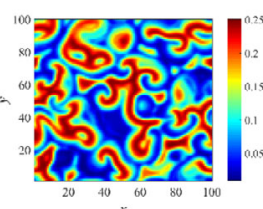

(b)

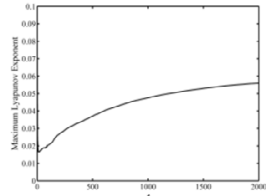

(f)

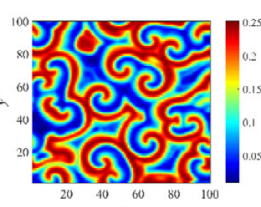

(c)

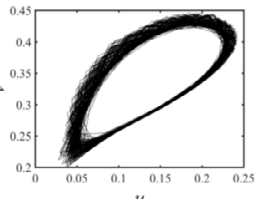

(g)

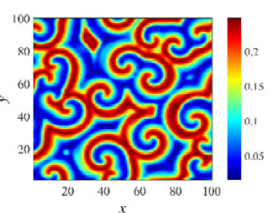

(d)

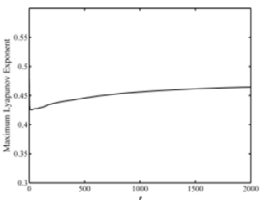

(h)

Figure 8 Self-organziation and nonlinear properties of a spiral pattern. (a)-(d) Evolution of the prey pattern at transient times $t=10, t=100, t=200$, and $t=500$, respectively; $(\mathbf{e})-(\mathbf{h})$ phase portraits and maximum Lyapunov exponent diagrams in the cases of uncoupling and coupling the population diffusion. The parameter values are given as $a=1.1, e_{1}=0.3, e_{2}=0.2, b=0.3739, \tau=3.59, \delta=1, h=8, n=100$

the gradual increase of time, a part of the spot and stripe patches in the pattern will disappear but some of them will combine together to form a circle (Fig. 7d). Comparing with the phase portraits and maximum Lyapunov exponent diagrams without and with consideration of the spatial diffusion (Fig. 7e-h), we find that the circle pattern is self-organized through the spatial symmetry breaking on a closed invariant curve. The pattern formation results to a transition from quasiperiodic behavior to chaotic behavior in the discrete predator-prey system. The self-organization of spiral pattern demonstrated in Fig. 8 is also induced by the Neimark-Sacker-Turing instability. Different from Fig. 7, this pattern emerges through spatial symmetry breaking on a state which is homogeneous in space and chaotic in time. As described in Fig. 8e-8h, the complexification of phase portrait and increase of maximum Lyapunov exponent imply that the pattern formation strengthens the chaotic state along the temporal scale. However, the distribution of the prey population still presents high degree of order in space. Such a result suggests that spatial order can generate from temporal disorder via the mechanism of Turing pattern formation. 


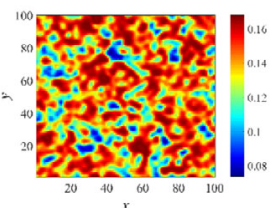

(a)

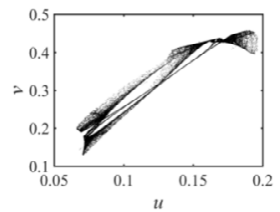

(e)

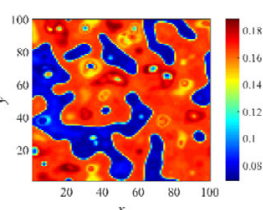

(b)

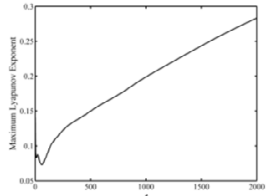

(f)

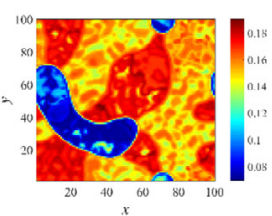

(c)

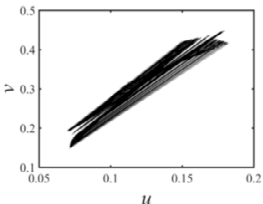

(g)

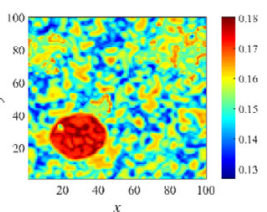

(d)

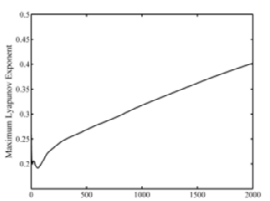

(h)

Figure 9 Self-organziation and nonlinear properties of a complex pattern induced by flip-Turing instability. (a)-(d) Evolution of the prey pattern at transient times $t=10, t=100, t=1000$, and $t=10,000$, respectively; (e)-(h) phase portraits and maximum Lyapunov exponent diagrams in the cases of uncoupling and coupling the population diffusion. The parameter values are given as $a=1.11, e_{1}=0.31, e_{2}=0.21, b=1.21, \tau=3.15$, $\delta=2, h=15, n=100$

When the discrete predator-prey system experiences flip bifurcation and Turing instability, the pattern formation can present a very complex situation different from above cases. As widely known, the flip bifurcation in discrete-time system often triggers a perioddoubling cascade and a route to chaos. Figure 9 exhibits a complex pattern which is selforganized by spatial symmetry breaking on the chaos induced by flip bifurcation. This pattern shows complicated configuration with almost no identified ordered structure in space and is always changing with time. As suggested by the comparison between Fig. 9e-f and Fig. $9 \mathrm{~g}-\mathrm{h}$, the discrete predator-prey system exhibits stronger chaos with the coupling of population diffusion.

It can be observed from the configuration in the above figures that a few patterns exhibit strong spatial autocorrelation, for example, in spot patterns, the cold or hot spots can occur repeatedly in space. Therefore, it deserves an investigation of the pattern characteristics from the aspect of spatial autocorrelation. In this research, the univariate Moran index is applied to quantify the spatial autocorrelation of the spatial patterns and the results are shown in Table 1. It is found that the patterns self-organized under the flip-Turing and the Neimark-Sacker-Turing instability demonstrate the highest spatial autocorrelation: larger than 0.9. This suggests that although the spatial distribution of prey patches in these pattern is irregular, the prey density in different patches may have small difference. Unsurprisingly, the regularity of structure in the patterns of Fig. 1 leads to relatively higher spatial autocorrelation, which is larger than 0.8. The spiral-spot and labyrinth patterns in Figs. 3 and 4 hold weak spatial autocorrelation, which verifies the spatiotemporal chaos and suggests large spatial variation in these patterns.

It is important to study the influence of neighborhood structure on the predator-prey dynamics and a comparison of pattern formation between the Moore and the von Neumann neighborhood structures needs to be made. The change of the neighborhood structure can influence the basic characteristics or even the type of self-organized patterns. Via comparison using the parametric conditions in Figs. 1, 7 and 8, we find the pattern types do not change with the neighborhood structures, but the patterns self-organized under Moore neighborhood structure seems like a zoom-in on that under the von Neumann 
Table 1 Univariate and bivariate Moran index for the simulated patterns

\begin{tabular}{lll}
\hline Patterns & Univariate Moran index & Bivariate Moran index \\
\hline Fig. 1(a) & 0.8195 & $2.6051 \times 10^{-4}$ \\
Fig. 1 (b) & 0.8037 & 0.0029 \\
Fig. 1(c) & 0.8056 & 0.0016 \\
Fig. 1(d) & 0.8071 & 0.0022 \\
Fig. 1(e) & 0.8078 & 0.0045 \\
Fig. 1(f) & 0.8424 & 0.0060 \\
Fig. 3 & -0.0397 & 0.0249 \\
Fig. 4 & -0.0588 & $9.3187 \times 10^{-4}$ \\
Fig. 5 & -0.2301 & $5.2240 \times 10^{-4}$ \\
Fig. 6(a) & -0.3217 & $8.3236 \times 10^{-4}$ \\
Fig. 6(b) & -0.2891 & $3.1231 \times 10^{-4}$ \\
Fig. 6(c) & -0.2301 & $5.2240 \times 10^{-4}$ \\
Fig. 7 & 0.9907 & 0.0733 \\
Fig. 8 & 0.9248 & 0.0277 \\
Fig. 9 & 0.9174 & 0.0136 \\
\hline
\end{tabular}

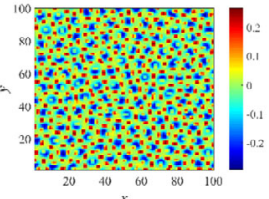

(a)

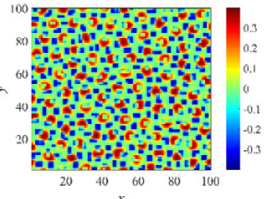

(b)

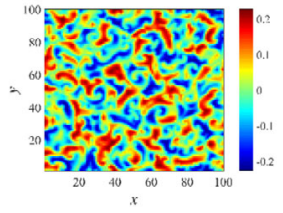

(c)

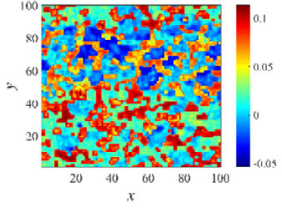

(d)

Figure 10 Subtraction between the two patterns which are simulated with the application of Moore and von Neumann neighborhood structures. Same initial conditions and parameter conditions are applied. The four cases are corresponding to (a) Fig. 1b, (b) Fig. 1f, (c) Fig. 8 and (d) Fig. 9, respectively

neighborhood structure, i.e., the spots, stripes and spirals enlarge about at four times. A comparison with the parametric conditions in Figs. 3-6 demonstrates that the Moore neighborhood leads to the formation of varying complex patterns, contrary to the very simple two-phase mosaic pattern and homogeneous state under von Neumann neighborhood.

Moreover, Fig. 10 is plotted with an exhibition of the subtraction between the patterns which are simulated with the application of Moore and von Neumann neighborhood structures. The results in Fig. 10 suggest that when the way of population dispersal changes, the pattern formation results in the predator-prey system may demonstrate great differences. Furthermore, we calculate the spatial correlation between the patterns self-organized under the two neighborhood structures via the method of bivariate Moran index [38-40], in order to quantitatively determine their differences. As shown in Table 1, all values of spatial correlation demonstrated by the bivariate Moran index are smaller than 0.1 and most of the values fluctuate around 0.001. These low values of the bivariate Moran index suggest the two neighborhood structures indeed lead to very large differences in the self-organized patterns.

With the pattern formation simulations, we find that limitations still exist in this research work. If the value of parameter $\tau$ is given making $\delta \tau / h^{2}>0.5$ or the initial conditions with large perturbations (for example, $u_{(x, y, 0)}=u_{0}(1+2(\varsigma-0.5))$ ) are provided, convergent results of patterns may not be obtained. Since the pattern formation can exhibit multistability under the flip-Turing and the Neimark-Sacker-Turing instability, the extrinsic stochastic spatiotemporal perturbations may geatly influence the pattern forma- 
tion results $[40,41]$. Also, the intrinsic stochasticity in pattern formation process is not considered.

\section{Discussion and conclusions}

The self-organization of patterns in predator-prey systems is a common phenomenon and has been widely investigated by researchers. Under the mechanism of spatial symmetry breaking driven by population diffusion, the predator-prey system may exhibit rich patterns for the coexistence between the predator and the prey. In previous research work, most of the spatiotemporal predator-prey models, regardless of continuous and discrete, are established and studied with the population diffusion based on the von Neumann neighborhood structure. However, the spatial population diffusion in real cases may be diverse and very complex. In this research, we explored the pattern formation of a discrete predator-prey system with the application of a CML model where the population diffusion is considered based on the Moore neighborhood structure. The results of pattern formation in this case as obtained in the present research can provide realistic significance in explaining a more general predator-prey coexistence.

With the application of stability analysis and Turing instability analysis, the pattern formation conditions for the discrete predator-prey system in this research are determined. Then, based on these conditions, numerical simulations reveal that the discrete predatorprey system can exhibit rich and complex patterns. We mainly focus on the pattern formation under three nonlinear mechanisms, the pure Turing instability, the Neimark-SackerTuring instability and the flip-Turing instability. The pure Turing instability can reproduce basic types of patterns as described in previous research, such as regular spot, stripe, labyrinth patterns and their intermediate patterns. This suggests the new model established in this research contains basic spatiotemporal dynamics of previous pattern formation models. The Neimark-Sacker-Turing and the flip-Turing instability can lead to the formation of circle, spiral and even more complex patterns, which are self-organized via spatial symmetry breaking on the states that are homogeneous in space and non-periodic in time. These patterns have the property of spatiotemporal chaos.

Little previous research work has investigated the pattern formation of spatiotemporally discrete predator-prey systems with the application of CML based on the von Neumann neighborhood structure, for example the investigations made by Petrovskii, Rodrigues and Mistro [5, 6, 21]. In comparison with their work, the study in this research presents a few differences or improvements. First, since the Moore neighborhood structure enhances the capability of population diffusion in the space, the predator-prey more easily reaches homogeneity than the case with the utilization of the von Neumann neighborhood structure and therefore the parameter domain for the Turing pattern formation shrinks. Second, under the pure Turing instability mechanism, we find the self-organization of two new spiral-spot and labyrinth patterns, which show the temporally oscillating and chaotic property. Comparing with the stationary patterns produced under von Neumann neighborhood structure, the dynamic properties of these oscillating patterns imply the predator and prey are quite active in space-time. Third, under the Neimark-Sacker-Turing instability, we find the formation of spiral patterns which occurs by spatial symmetry breaking on a state that is homogeneous in space and chaotic in time. The emergence of such spiral pattern suggests that spatial order can emerge from temporal disorder. It can be inferred that when the predator-prey dynamics on one site is chaotic, the spatially global dynamics 
may still be predictable. Fourth, the flip-Turing instability and the corresponding pattern formation are further studied. The simulated patterns shows complicated configuration with almost no identified ordered structure in space, suggesting stronger chaos possibly due to the coupling of population diffusion under the Moore neighborhood structure.

The investigation in the present research, from the viewpoint of mathematical modeling and simulation, reveals the importance of the way of population dispersal in the spatiotemporal dynamics of predator-prey systems. Compared with the von Neumann neighborhood structure under the same parametric conditions, the usage of the Moore neighborhood can change the pattern formation mainly in two aspects: first, changing the pattern types and increasing the complexity of patterns; second, enlarging the prey patches such as cold or hot spots, stripes and spirals. Moreover, as quantitatively determined by the bivariate Moran index, the patterns self-organized under the two neighborhood structures show weak spatial correlation, suggesting great differences.

In recent research work, the usage of von Neumann neighborhood structure for pattern simulation is common. This may result from the von Neumann neighborhood showing a direct difference form of the classical continuous Laplacian operator. From the ecological aspect, the application of the Moore neighborhood structure suggests a case of population diffusion which extends to all sites around by arbitrary ways and therefore is less restrained in space. In the research of cellular automata, the Moore neighborhood is once used to simulate the dynamics in game of life [42, 43]. Moreover, if we consider the population diffusion among different habitat patches as complex network, the von Neumann and Moore neighborhoods describe two different network structures. This suggests both neighborhood structures can be important for the study of the pattern formation.

Since the change of neighborhood structure may lead to a totally different pattern formation, in the establishment of spatiotemporal models for predator-prey systems, how the populations diffuse in the space should be identified with care. Also, the research work on the predator-prey systems where the population diffusion occurs based on different neighborhood structures deserves further investigation in the future.

\section{Acknowledgements}

The authors would like to acknowledge with great gratitude the support of all funders.

Funding

National Natural Science Foundation of China (No. 11802093), National Water Pollution Control and Treatment Science and Technology Major Project (No. 2017ZX07101-002), and Fundamental Research Funds for the Central Universities (No. JB2017069).

Availability of data and materials

The datasets used and/or analysed during the current study are available from the corresponding author on request.

Ethics approval and consent to participate

Not applicable.

Competing interests

The authors have no financial and non-financial competing interests for this research work.

Consent for publication

The authors agree to publish the paper based on consensus once it is accepted. 


\section{Publisher's Note}

Springer Nature remains neutral with regard to jurisdictional claims in published maps and institutional affiliations.

\section{Received: 4 December 2018 Accepted: 4 September 2019 Published online: 18 September 2019}

\section{References}

1. Kondo, S., Miura, T.: Reaction-diffusion model as a framework for understanding biological pattern formation. Science 329(5999), 1616-1620 (2010)

2. Xu, J., Yang, G., Xi, H., Su, J.: Pattern dynamics of a predator-prey reaction-diffusion model with spatiotemporal delay. Nonlinear Dyn. 81(4), 2155-2163 (2015)

3. Brigatti, E., Oliva, M., Núñez-López, M., Oliveros-Ramos, R., Benavides, J.: Pattern formation in a predator-prey system characterized by a spatial scale of interaction. Europhys. Lett. 88(6), 68002 (2009)

4. Li, C.: Spatiotemporal pattern formation of an invasion-diffusion predator-prey system. J. Syst. Sci. Math. Sci. 37(3), 950-964 (2017)

5. Mistro, D.C., Rodrigues, L.A.D., Petrovskii, S.: Spatiotemporal complexity of biological invasion in a space- and time-discrete predator-prey system with the strong Allee effect. Ecol. Complex. 9, 16-32 (2012)

6. Rodrigues, L.A.D., Mistro, D.C., Petrovskii, S.: Pattern formation in a space- and time-discrete predator-prey system with a strong Allee effect. Theor. Ecol. 5(3), 341-362 (2012)

7. Punithan, D., Kim, D.K., McKay, R.I.B.: Spatio-temporal dynamics and quantification of daisyworld in two-dimensional coupled map lattices. Ecol. Complex. 12, 43-57 (2012)

8. Czarnecka, B.: Spatiotemporal patterns of genets and ramets in a population of clonal perennial senecio rivularis: plant features and habitat effects. Ann. Bot. Fenn. 45(1), 19-32 (2008)

9. Krekhov, A., Dressel, B., Pesch, W., Delev, V., Batyrshin, E.: Spatiotemporal complexity of electroconvection patterns in nematic liquid crystals. Phys. Rev. E, Stat. Nonlinear Soft Matter Phys. 92(6-1), 062510 (2015)

10. Nandy, A.S., Mitchell, J.F., Jadi, M.P., Reynolds, J.H.: Neurons in macaque Area V4 are tuned for complex spatiotempora patterns. Neuron 91(4), 920-930 (2016)

11. Kirkwood, J.G., Oppenheim, I.: Chemical Thermodynamics, 1st edn. McGraw-Hill, New York (1961)

12. Turing, A.M.: The chemical basis of morphogenesis. Philos. Trans. R. Soc. Lond. B, Biol. Sci. 237, 37-72 (1952)

13. Ouyang, Q., Swinney, H.L.: Transition from a uniform state to hexagonal and striped Turing patterns. Nature 352(6336), 610-612 (1991)

14. Petrov, V., Qi, O., Swinney, H.L.: Resonant pattern formation in a chemical system. Nature 388(6643), 655-657 (1997)

15. Wang, J., Wei, J., Shi, J.: Global bifurcation analysis and pattern formation in homogeneous diffusive predator-prey systems. J. Differ. Equ. 260(4), 3495-3523 (2016)

16. Huang, T., Zhang, H., Yang, H., Wang, N., Zhang, F.: Complex patterns in a space- and time-discrete predator-prey model with Beddington-DeAngelis functional response. Commun. Nonlinear Sci. Numer. Simul. 43, 182-199 (2017)

17. Huang, T., Zhang, H., Yang, H.: Spatiotemporal complexity of a discrete space-time predator-prey system with selfand cross-diffusion. Appl. Math. Model. 47, 637-655 (2017)

18. May, R.M.: Simple mathematical models with very complicated dynamics. Nature 261(5560), 459-467 (1976)

19. Kaneko, K.: Pattern dynamics in spatiotemporal chaos: pattern selection, diffusion of defect and pattern competition intermittency. Phys. D, Nonlinear Phenom. 34(1), 1-41 (1989)

20. Kaneko, K.: Period-doubling of kink-antikink patterns, quasiperiodicity in antiferro-like structures and spatial intermittency in coupled logistic lattice towards a prelude of a "field theory of chaos". Prog. Theor. Phys. 72(3), 480-486 (1984)

21. Rodrigues, L.A.D., Mistro, D.C., Petrovskii, S.: Pattern formation in a space- and time-discrete predator-prey system with a strong Allee effect. Theor. Ecol. 5(3), 341-362 (2012)

22. Huang, T., Zhang, H.: Bifurcation, chaos and pattern formation in a space- and time-discrete predator-prey system. Chaos Solitons Fractals 91, 92-107 (2016)

23. Kaneko, K.: Coupled map lattice. In: Chaos, Order and Patterns, Lake Como, 1990. NATO Adv. Sci. Inst. Ser. B Phys, vol. 280, pp. 237-247. Plenum, New York (1991)

24. Yin, H., Xiao, X., Wen, X., Liu, K.: Pattern analysis of a modified Leslie-Gower predator-prey model with Crowley-Martin functional response and diffusion. Comput. Math. Appl. 67(8), 1607-1621 (2014)

25. Liu, M., Du, C., Deng, M.: Persistence and extinction of a modified Leslie-Gower Holling-type Il stochastic predator-prey model with impulsive toxicant input in polluted environments. Nonlinear Anal. Hybrid Syst. 27, 177-190 (2018)

26. Singh, M.K., Bhadauria, B.S., Singh, B.K.: Bifurcation analysis of modified Leslie-Gower predator-prey model with double Allee effect. Ain Shams Eng. J. 9(4), 1263-1277 (2018)

27. Aziz-Alaoui, M.A., Daher-Okiye, M.:. Boundedness and global stability for a predator-prey model with modified Leslie-Gower and Holling type II shemes. Appl. Math. Lett. 16, 1069-1075 (2003)

28. Nindjin, A.F., Aziz-Alaoui, M.A.: Persistence and global stability in a delayed Leslie-Gower type three species food chain. J. Math. Anal. Appl. 340(1), 340-357 (2008)

29. Nindjin, A.F., Aziz-Alaoui, M.A., Cadivel, M.: Analysis of a predator prey model with modified Leslie-Gower and Holling-type II schemes with time delay. Nonlinear Anal., Real World Appl. 7(5), 1104-1118 (2006)

30. Zhang, F., Zhang, H., Ma, S., Meng, T., Huang, T., Yang, H.: Self-organized patterns induced by Neimark-Sacker, flip and Turing bifurcations in a discrete predator-prey model with Lesie-Gower functional response. Entropy 19(6), 258 (2017)

31. Sun, G., Sarwardi, S., Pal, P.J., Rahman, Md.S.: The spatial patterns through diffusion-driven instability in modified Leslie-Gower and Holling-type II predator-prey model. J. Biol. Syst. 18, 593-603 (2010)

32. Aziz-Alaoui, M.A., Okiye, M.D.: Boundedness and global stability for a predator-prey model with modified Leslie-Gower and Holling-type II schemes. Appl. Math. Lett. 16(7), 1069-1075 (2003)

33. Aziz-Alaoui, M.A., Okiye, M.D.: Boundedness and global stability for a predator-prey model with modified Leslie-Gower and Holling-type II schemes. Appl. Math. Lett. 16(7), 1069-1075 (2003)

34. Tian, Y., Weng, P.: Stability analysis of diffusive predator-prey model with modified Leslie-Gower and Holling-type II schemes. Acta Appl. Math. 114(3), 173-192 (2011) 
35. Neubert, M.G., Caswell, H., Murray, J.D.: Transient dynamics and pattern formation: reactivity is necessary for Turing instabilities. Math. Biosci. 175(1), 1-11 (2002)

36. Huang, T., Zhang, H., Cong, X., Pan, G., Zhang, X., Liu, Z.: Exploring spatiotemporal complexity of a predator-prey system with migration and diffusion by a three-chain coupled map lattice. Complexity 2019, 3148323 (2019)

37. Banerjee, M.: Comments on "L. N. Guin, M. Haque, P. K. Mandal, The spatial patterns through diffusion-driven instability in a predator-prey model, Appl. Math. Model. 36 (2012) 1825-1841.." Appl. Math. Model. 39, 297-299 (2015)

38. Robertson, C., Mazzetta, C., D'Onofrio, A.: Regional variation and spatial correlation. In: Boyle, P., Smans, M. (eds.) Atlas of Cancer Mortality in the European Union and the European Economic Area 1993-1997, pp. 91-113, Chap. 5 (2008)

39. D'Onofrio, A., Mazzetta, C., Robertson, C., Smans, M., Boyle, P., Boniol, M.: Maps and atlases of cancer mortality: a review of a useful tool to trigger new questions. Ecancermedicalscience 10, 670 (2016)

40. De Franciscis, S., D'Onofrio, A.: Spatiotemporal bounded noises and transitions induced by them in solutions of the real Ginzburg-Landau model. Phys. Rev. E 86(2), 021118 (2012)

41. De Franciscis, S., D'Onofrio, A.: Spatio-temporal sine-Wiener bounded noise and its effect on Ginzburg-Landau model. Nonlinear Dyn. 74(3), 607-613 (2013)

42. Conway, J.: The game of life. Sci. Am. 223(4), 4 (1970)

43. Wolfram, S.: Theory and Applications of Cellular Automata. World Scientific, Singapore (1986)

\section{Submit your manuscript to a SpringerOpen ${ }^{\circ}$ journal and benefit from:}

- Convenient online submission

- Rigorous peer review

- Open access: articles freely available online

- High visibility within the field

- Retaining the copyright to your article

Submit your next manuscript at $\mathbf{s p r i n g e r o p e n . c o m ~}$ 